\title{
Convex Drawings of 3-Connected Plane Graphs
}

Extended Abstract

\author{
Nicolas Bonichon ${ }^{1}$, Stefan Felsner ${ }^{2}$, and Mohamed Mosbah ${ }^{1}$ \\ 1 LaBRI , Université Bordeaux-1, \\ 351, cours de la Libération, 33405 Talence Cedex, France \\ \{bonichon, mosbah\}@labri.fr \\ 2 Technische Universität Berlin, Institut für Mathematik, MA 6-1, \\ Strasse des 17. Juni 136, 10623 Berlin, Germany \\ felsner@math.tu-berlin.de
}

\begin{abstract}
We use Schnyder woods of 3-connected planar graphs to produce convex straight line drawings on a grid of size $(n-2-\Delta) \times(n-2-\Delta)$. The parameter $\Delta \geq 0$ depends on the the Schnyder wood used for the drawing. This parameter is in the range $0 \leq \Delta \leq \frac{n}{2}-2$.
\end{abstract}

\section{Introduction}

We investigate crossing-free straight-line drawings of planar graphs with the restriction that the vertices of the graph have to be located at integer grid points. The aim is to keep the area of an axis-aligned rectangle which covers the complete drawing as small as possible. It is known that a square of side-length $n-2$, i.e., a $(n-2) \times(n-2)$ grid is enough to host every planar graph.

A drawing with the property that the boundary of every face (including the outer face) is a convex polygon is called a convex drawing. Convex drawings exist for every 3-connected planar graph. Again the aim is to keep the area of such a drawing as small as possible.

It is important to distinguish between convex drawings and strictly convex drawings. A drawing is strictly convex if every interior angle is less than $180^{\circ}$ and every outer angle greater than $180^{\circ}$. In this paper we deal with convex drawings. The grid size for strictly convex drawings was recently studied by Rote [1], he proves that an $O\left(n^{7 / 3}\right) \times O\left(n^{7 / 3}\right)$ grid is enough for strictly convex drawings of planar graphs with $n$ vertices.

The question whether every planar graph has a straight line embedding on a grid of polynomial size was raised by Rosenstiehl and Tarjan [2]. Unaware of the problem Schnyder [3] constructed a barycentric representation which immediately translates to an embedding on the $(2 n-6) \times(2 n-6)$ grid. The first explicit answer to the question was given by de Fraysseix, Pach and Pollack $[4,5]$. They construct straight line embeddings on an $(2 n-4) \times(n-2)$ grid and show that the embedding can be computed in $O(n \log n)$. De Fraysseix et al. also observed a lower bound of $\left(\frac{2}{3} n-1\right) \times\left(\frac{2}{3} n-1\right)$ for grid embeddings of the $n$ vertex graph containing a nested sequence of $n / 3$ triangles. It is conjectured that this is the worst case, i.e., that every planar graph can be embedded on the 
$\left(\frac{2}{3} n-1\right) \times\left(\frac{2}{3} n-1\right)$ grid. 4-connected planar graphs with at least four vertices on the outer face can be drawn even more compactly. Work of He [6] and Miura et al. [7] shows that these graphs can be embedded on the $\frac{n}{2} \times \frac{n}{2}$ grid.

In his second paper Schnyder proves the existence of an embedding on the $(n-2) \times(n-2)$ grid which can be computed in $O(n)$ time. In general Schnyder's result from [8] is still unbeaten. Lately, Zhang and He [9] used the minimum Schnyder wood of a triangulation to prove a bound of $\left(n-1-\Delta^{\cap}\right) \times\left(n-1-\Delta^{\cap}\right)$, where $\Delta^{\circledR}$ is the number of cyclic faces in the minimum Schnyder wood.

Though it is implicitly contained in Steinitz's characterization of 3-connected planar graphs as the skeleton graphs of 3-dimensional polytopes the existence of convex drawings for these graphs is known as Tutte's theorem. The idea for Tutte's proof $[10,11]$ is known as spring-embedding. Technically the embedding is obtained as solution to a system of linear equations. Kant [12] has extended the approach of de Fraysseix et al. to construct convex drawings on the $(2 n-4) \times(n-$ $2)$ grid. The grid size was reduced to $(n-2) \times(n-2)$ by Chrobak and Kant [13]. Schnyder and Trotter [14] have worked on ideas of using Schnyder woods for convex grid embeddings. The basic approach was independently worked out by di Battista et al. [15] and Felsner [16] this results in convex grid drawings on the $(f-1) \times(f-1)$ grid, where $f$ is the number of faces of the drawing. In this paper this basic algorithm is used but the size of the required grid is reduced by some new ideas. Loosely speaking some edges are eliminated which results in the reduction of $f$. This can be done until at most $n-\Delta$ faces remain. The eliminated edges can be reinserted in the resulting drawing on the $(n-1-\Delta) \times(n-1-\Delta)$ grid, with $\Delta \geq 0 . \Delta \geq n-f$. The drawing procedure can be implemented to run in linear time. The algorithm has been implemented and integrated in PIGALE library ${ }^{1}$.

In the next section we introduce Schnyder woods. It is shown how to use Schnyder woods to obtain convex drawings of 3-connected planar maps. The lattice of Schnyder woods is discussed and a new operation called merge is introduced as a tool for transforming Schnyder woods and their underlying graphs.

Section 3 contains the generic drawing algorithm. It is shown that this algorithm produces convex drawings and the size of the grid required for the drawing is analyzed. The main ingredient of this analysis is a bound on the number of merges applicable to a Schnyder wood. In particular it is shown that starting with the Schnyder wood of a triangulation a sequence of $n-4+\Delta^{\oplus}-\Delta^{+r}$ merge operations is admissible.

Section 4 presents a technique to decrease of the side-length of the grid by one. This small reduction, however, is crucial to match Schnyder's $(n-2) \times(n-2)$ bound for planar triangulations.

\section{Schnyder Woods}

Schnyder defined special colorings and orientations of the inner edges of a triangulation. In [3] and [8] he applied these Schnyder woods to characterize planar

\footnotetext{
${ }^{1}$ http://pigale. sourceforge.net
} 
graphs and to draw planar graphs on small grid sizes. Here we describe a generalization of Schnyder woods for 3-connected planar graphs. Such generalizations have been obtained in [15] and [16], in our exposition we follow [17].

A planar map $M$ is a simple planar graph $G$ together with a fixed planar embedding of $G$ in the plane. A suspension $M^{\sigma}$ of $M$ is obtained as follows: Three different vertices from the outer face of $M$ are specified and named $a_{1}, a_{2}, a_{3}$ in clockwise order. (For ease of visualization we identify the indices 1,2,3 with colors red, green, blue). At each of the three special vertices $a_{i}$, called suspension vertices, a half-edge reaching into the outer face is attached.

Let $M^{\sigma}$ be a suspension of a planar map. A Schnyder wood is an orientation and coloring of the edges of $M^{\sigma}$ with the colors $1,2,3$ satisfying the following rules.

(W1) Every edge $e$ is oriented by one or two opposite directions. The directions of edges are colored such that if $e$ is bi-directed the two directions have distinct colors.

(W2) The half-edge at $a_{i}$ is directed outwards and colored $i$.

(W3) Every vertex $v$ has outdegree one in each color. The edges $e_{1}, e_{2}, e_{3}$ leaving $v$ in colors 1,2,3 occur in clockwise order. Each edge entering $v$ in color $i$ enters $v$ in the clockwise sector from $e_{i+1}$ to $e_{i-1}$. See Figure 1 .

(W4) There is no interior face whose boundary is a directed cycle in one color.

Fact 1 There is a Schnyder wood for $M^{\sigma}$, if $M^{\sigma}$ is the suspension $M^{\sigma}$ of a 3-connected planar map. Actually, a Schnyder wood for $M^{\sigma}$ exists under the weaker condition that the graph obtained by adding a new vertex $v_{\infty}$ as the second endpoint for the three half-edges is planar and 3-connected.

Given a Schnyder wood, let $T_{i}$ be the set of edges colored $i$ with the direction they have in this color. Since every inner vertex has outdegree one in $T_{i}$ every $v$ is the starting vertex of a unique $i$-path $P_{i}(v)$ in $T_{i}$.

Fact 2 The digraph $T_{i}$ is acyclic, even more, $T_{i}$ is a tree with root $a_{i}$.

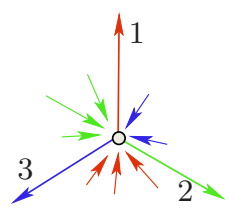

(a)

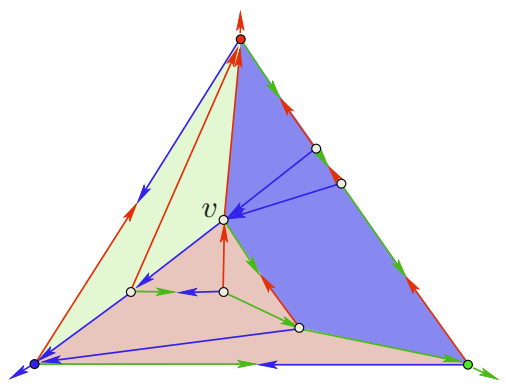

(b)

Fig. 1. (a) Edge colorings ${ }^{2}$ and orientations at a vertex. (b) A Schnyder wood and the regions of vertex $v$.

\footnotetext{
${ }^{2}$ If you can't see the colors look up the colorful electronic versions at the authors homepages.
} 


\subsection{Convex Drawings via Face-Counting}

Schnyder and Trotter [14] had some ideas of using Schnyder woods for convex grid embeddings. The approach has been worked out in [15] and [16]. We describe the technique omitting some details.

From the vertex condition (W3) it follows that for $i \neq j$ the paths $P_{i}(v)$ and $P_{j}(v)$ have $v$ as the only common vertex. Therefore, $P_{1}(v), P_{2}(v), P_{3}(v)$ divide $M$ into three regions $R_{1}(v), R_{2}(v)$ and $R_{3}(v)$, where $R_{i}(v)$ denotes the region bounded by and including the two paths $P_{i-1}(v)$ and $P_{i+1}(v)$, see Fig. 1 .

Fact 3 (a) $R_{i}(u) \subseteq R_{i}(v)$ iff $u \in R_{i}(v)$.

(b) $R_{i}(u)=R_{i}(v)$ iff there $i s$ a path of bicolored edges in colors $i-1$ and $i+1$ connecting $u$ and $v$.

(c) For all $u$, $v$ there are $i$ and $j$ with $R_{i}(u) \subset R_{i}(v)$ and $R_{j}(v) \subset R_{j}(u)$.

The face-count of a vertex $v$ is the vector $\left(v_{1}, v_{2}, v_{3}\right)$, where $v_{i}$ is the number of faces of $M$ contained in region $R_{i}(v)$.

Fact 4 For every edge $\{u, w\}$ and vertex $v \neq u, w$ there is a color $i$ with $\{u, w\} \in$ $R_{i}(v)$, hence, $u_{i} \leq v_{i}$ and $w_{i} \leq v_{i}$.

Inclusion properties of the three regions of adjacent vertices imply:

Fact 5 (a) If edge $(u, v)$ is uni-directed in color $i$, then

$$
u_{i}<v_{i}, u_{i-1}>v_{i-1} \text { and } u_{i+1}>v_{i+1} \text {. }
$$

(b) If $(u, v)$ is directed in color $i-1$ and $(v, u)$ in color $i+1$, then

$u_{i}=v_{i}, u_{i-1}>v_{i-1}$ and $u_{i+1}<v_{i+1}$.

Clearly, each vertex $v$ has $v_{1}+v_{2}+v_{3}=f-1$, where $f$ is the number of faces of $M$. Hence, we have a mapping of the vertices of the graph to the plane $T_{f}=\left\{\left(x_{1}, x_{2}, x_{3}\right): x_{1}+x_{2}+x_{3}=f-1\right\}$ in $\mathbb{R}^{3}$. Connecting the points corresponding to adjacent vertices by the line segment between them yields a drawing $\mu(M)$ of $M$ in the plane $T_{f}$.

Color and orientation of edges are nicely encoded in this drawing: Let $v$ be a vertex with $\mu(v)=\left(v_{1}, v_{2}, v_{3}\right)$. The three lines $x_{1}=v_{1}, x_{2}=v_{2}$ and $x_{3}=v_{3}$ partition the plane $T_{f}$ into six wedges with apex $\mu(v)$. By Fact 5 the color and orientation of edges incident to $v$ is determined by the wedge containing them, see Figure 2. In particular the bicolored edges are the edges supported by the lines defining the wedges.

Theorem 1. The drawing $\mu(M)$ is a convex drawing of $M$ in $T_{f}$. Dropping the third coordinate yields a convex drawing of $M$ on the $(f-1) \times(f-1)$ grid.

\subsection{The Lattice of Schnyder Woods}

In general the suspension $M^{\sigma}$ of a 3-connected planar map will admit many Schnyder woods. Felsner [18] has shown that the set of all Schnyder woods of a given $M^{\sigma}$ has the structure of a distributive lattice. As we will make use of some elements of this theory we recall some definitions and the main results.

Think of the three half-edges of $M^{\sigma}$ as noncrossing infinite rays. These rays partition the outer face of $M$ into three parts. The suspension dual $M^{\sigma^{*}}$ of $M^{\sigma}$ 


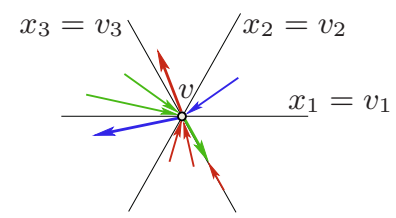

Fig. 2. Wedges and edges at a vertex $v$ in the plane $T_{f}$.

is the dual of this map. Thus $M \sigma^{*}$ has a triangle $b_{1}, b_{2}, b_{3}$ corresponding to the unbounded face of $M$.

The completion $\widetilde{M^{\sigma}}$ of a plane suspension $M^{\sigma}$ and its dual $M^{\sigma^{*}}$ is obtained as follows: Superimpose $M^{\sigma}$ and $M^{\sigma^{*}}$ so that exactly the primal dual pairs of edges cross (the half edge at $a_{i}$ has a crossing with the dual edge $\left\{b_{j}, b_{k}\right\}$, for $\{i, j, k\}=\{1,2,3\})$. At each crossing place a new vertex such that this new edge vertex is subdividing the two crossing edges.

The completion $\widetilde{M^{\sigma}}$ is planar, every edge-vertex has degree four and there are six half-edges reaching into the unbounded face.

A 3-orientation of the completion $\widetilde{M^{\sigma}}$ of $M^{\sigma}$ is an orientation of the edges of $\widetilde{M^{\sigma}}$ such that:

(O1) outdeg $(v)=3$ for all primal- and dual-vertices $v$.

$(\mathrm{O} 2) \operatorname{indeg}\left(v_{e}\right)=3$ for all edge-vertices $v_{e}$ (hence, $\operatorname{outdeg}\left(v_{e}\right)=1$ ).

(O3) All half-edges are out-edges of their vertex.

Theorem 2. Let $M^{\sigma}$ be a suspension of a 3-connected plane graph $M$. The following structures are in bijection: Schnyder woods of $M^{\sigma}$, Schnyder woods of the suspension dual $M^{\sigma^{*}}$ and 3-orientations of the completion $\widetilde{M^{\sigma}}$.

The bijections are illustrated in Figure 3.

The lattice structure of Schnyder woods is best understood by looking at 3-orientations: Let $X$ be a 3-orientation and let $C$ be directed cycle of a $X$. Reverting the orientation of all edges of $C$ yields another 3 -orientation $X^{C}$. If $C$ is a simple cycle it has a connected interior an we can speak of the clockwise and the counterclockwise order of $C$. Define $X \succ X^{C}$ if $C$ is a clockwise cycle in $X$. The transitive closure $\succ^{*}$ of this relation is an order relation on the set of 3-orientations.
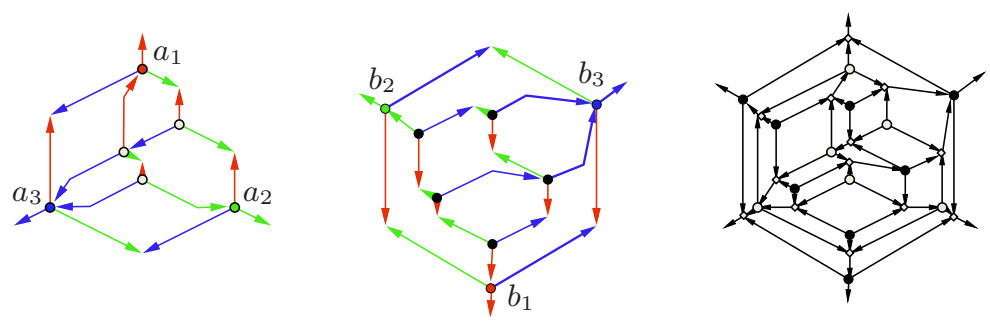

Fig. 3. The bijections for Theorem 2. 
Theorem 3. The relation $\succ^{*}$ is the order relation of a distributive lattice on the set of 3-orientations of the completion $\widetilde{M^{\sigma}}$ of a suspension $M^{\sigma}$ of a 3-connected planar map. The unique minimum 3-orientation contains no clockwise directed cycles.

In view of Theorem 2 a suspension $M^{\sigma}$ has unique minimum Schnyder wood $S_{\text {Min }}$. Figure 4 shows two sub-structures which are impossible in $S_{\text {Min }}$ : an unidirected edge incoming at $v$ in color $i+1$ such that the counterclockwise next edge is bi-directed, outgoing at $v$ in color $i-1$ and incoming in color $i$; and a clockwise triangle of uni-directed edges, such a triangle must have colors $i, i+1$, $i+2$ in this clockwise order.

Theorem 4. ([19]) Let $G$ be a 3-connected plane graph. The minimal Schnyder Wood $S_{\mathrm{Min}}$ of $G$ can be computed in linear time.
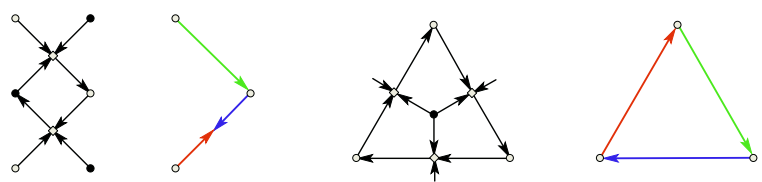

Fig. 4. Two types of clockwise cycles in 3-orientations and the corresponding substructures of Schnyder woods.

\subsection{Merging and Splitting}

The operations merge and split introduced in this section operate on Schnyder woods and the underlying graph. Merge and split can be seen as inverse operations, corresponding to the deletion and insertion of an edge.

Given a Schnyder wood, a knee at vertex $v$ is a pair of uni-directed edges adjacent at an angle of $v$ such that one of the edges is incoming and the other outgoing at $v$. Knees come in two kinds, if the in-edge of the knee is the clockwise neighbor of the out-edge at $v$ we speak of a a $c w$-knee, otherwise, if the in-edge of the knee is the counterclockwise neighbor of the out-edge it is a ccw-knee.

Let $(u, v),(v, w)$ be a knee at $v$. Suppose that the color of $(v, w)$ is $i$ by the vertex condition the color of $(u, v)$ is $i-1$ if it is a cw-knee and $i+1$ if it is a ccw-knee. The merge of the knee consist of the deletion of the out-edge $(v, w)$ while making $(u, v)$ a bi-directed edge outgoing at $v$ in color $i$ and incoming in the same color as before. Depending on the type of the knee we distinguish between clockwise and counterclockwise merge operations. Figure 5 illustrates the definition.

Lemma 1. Let $S$ be a Schnyder wood, the coloring and orientation of edges after merging a knee is again a Schnyder wood.

A split of a bi-directed edge is the inverse operation of a merge. In the context of this paper we only need one very specific type of split. The short cw-split is the inverse of a cw-merge with the additional property that $(u, w)$ is an edge, i.e., $u, v, w$ is a triangle. 


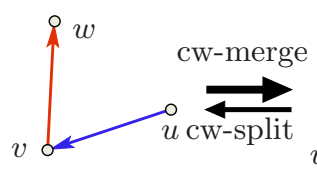

${ }^{\circ} w$
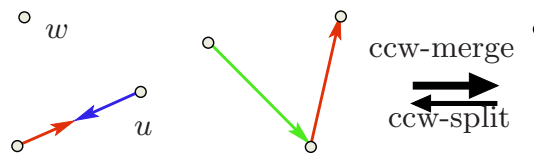

Fig. 5. Clockwise and counterclockwise merge and split.

\section{The Drawing Algorithm}

Let $M$ be a 3-connected planar map with $n$ vertices and $f$ faces. The steps of the drawing algorithm with input $M$ are the following:

(A1) Choose three vertices from the outer face for the suspension $M^{\sigma}$.

(A2) Compute the minimum Schnyder wood $S_{\text {Min }}$ for $M^{\sigma}$ and let $S_{0}=S_{\text {Min }}$.

(A3) Compute a maximal cw-merge sequence $S_{0} \rightarrow S_{1} \rightarrow \ldots S_{k}$ of Schnyder woods, i.e., $S_{i+1}$ is obtained from $S_{i}$ by a cw-merge and $S_{k}$ contains no cw-knee.

(A4) Use face-counting to draw $S_{k}$ on the $(f-k-1) \times(f-k-1)$ grid.

(A5) Reinsert all edges which have been deleted by merge operations into the drawing from the previous step.

With Figure 6 we illustrate step A3 of the algorithm.

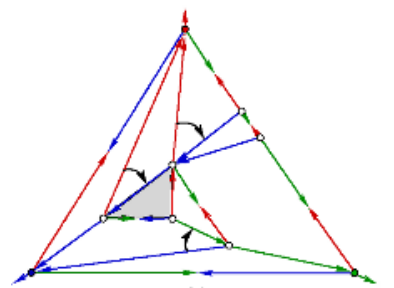

(a)

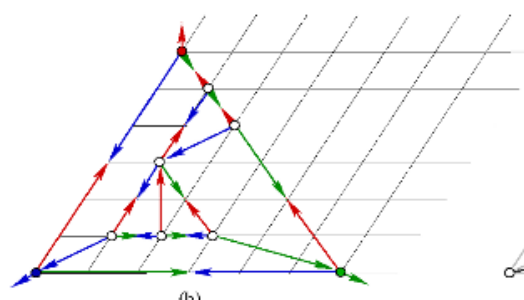

(b)

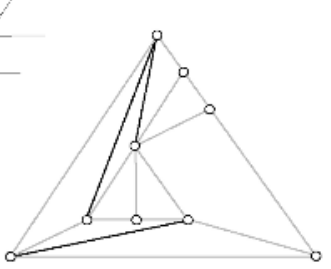

(c)

Fig. 6. (a) A Schnyder wood $S_{0}$, cw-knees are indicated by arcs. (b) The final Schnyder wood of a merge sequence. (c) The example graph with $n=f=9$ drawn on the $6 \times 6$ grid.

\subsection{The Drawing Is Convex}

Theorem 5. Reinserting all the edges which have been deleted by a sequence of cw-merge operations into the drawing of $S_{k}$ obtained in A4 keeps the drawing planar and convex.

The drawing steps of the algorithm (A4 and A5) are illustrated in Figure 6. Essential for the proof of the theorem is the following lemma:

Lemma 2. Given a Schnyder wood of a suspended map $M^{\sigma}$ and let $F$ be an interior face. The orientation and color of edges around $F$ obeys the following rule (see Figure 7): 

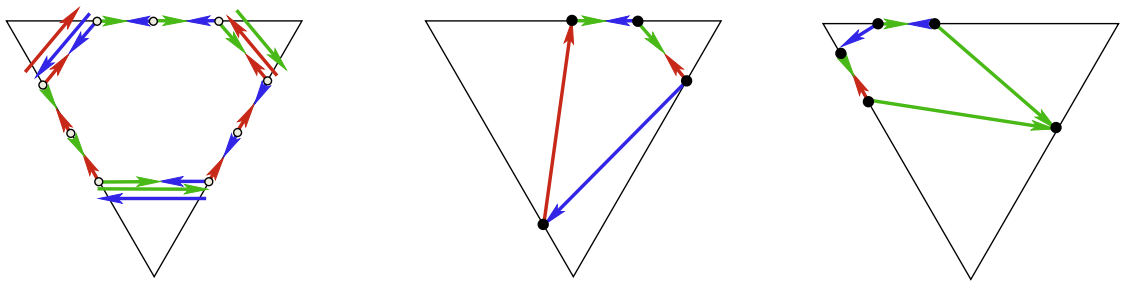

Fig. 7. The generic structure of a face as described by Lemma 2 and two concrete instances.

- In clockwise order the types of edges at the boundary of the face can be described as follows (in case of bi-directed edges the clockwise color is noted first): One edge from the set $\{$ red-cw, blue-ccw, red-blue\}, any number (may be 0) of edges green-blue, one edge from the set \{green-cw, red-ccw, greenred\}, any number of edges blue-red, one edge from the set \{blue-cw, green$\mathrm{ccw}$, blue-green\}, any number of edges red-green.

\subsection{The Number of Merges}

Essential for the grid-size required for the drawing produced by the algorithm is the length $k$ of the merge sequence computed in step A3. The main result in this subsection is a lower bound for $k$ in terms of easily recognizable substructures of the initial Schnyder wood $S$ computed in step A2 of the algorithm.

As a warm-up let us consider the case where $M$ is a triangulation and $S$ is an arbitrary Schnyder wood of $M$. Consider the $(2 n-4)-4$ triangles of $S$ which are bounded by three uni-directed edges. These triangles can be partitioned into two classes: Class one are those with at least two clockwise oriented edges on the boundary and class two are those with at least two counterclockwise edges on the boundary. Suppose that the number $C_{1}$ of triangles of class one is the larger one, i.e., $C_{1} \geq n-4 \geq C_{2}$. In a triangle $T$ of class one there is a knee of two consecutive clockwise edges of $T$, this knee is a candidate for a clockwise merge. Since every edge is clockwise only for one of its neighboring triangles these $C_{1}$ merges can be performed independently. It follows that starting from $S$ there is a merge sequence of length $k \geq C_{1} \geq n-4$. This estimate yields drawing of triangulations on grids of size at most $(f-(n-4)-1) \times(f-(n-4)-1)=(n-1) \times(n-1)$.

The following proposition gives a better result.

Proposition 1. Let $S$ be a Schnyder wood with $\Delta_{S}^{+r}$ clockwise and $\Delta_{S}^{\oplus}$ counterclockwise triangles. The number of cw-merges applicable in a merge sequence starting with $S$ is at least $n-4-\Delta_{S}^{+r}+\Delta_{S}^{\bullet}$.

To estimate the number of merges that can be applied to a Schnyder wood $S$ of a non-triangulated map we need more terminology. Let $\Delta_{S}^{\bullet}$ be the number of faces, with a counterclockwise edge in each of the three colors and not adjacent to a suspension vertex. These edges do not need to be uni-directed. 
$\Delta_{S}^{+}$counts the number of clockwise triangles of uni-directed edges together with patterns of the following type: an uni-directed edge incoming at $v$ in color $i+1$ such that the counterclockwise next edge is bi-directed, outgoing at $v$ in color $i-1$ and incoming in color $i$ (see Figure 4).

Theorem 6. Let $S$ be a Schnyder wood of a 3-connected planar map. The number of cw-merges that can be applied to $S$ is at least $f-n+\Delta^{-}-\Delta^{+}$.

Given an arbitrary Schnyder wood the contribution of $\Delta^{\oplus}-\Delta^{+}$in the above formula may well be negative. However, the choice of $S=S_{\text {Min }}$ guarantees that $\Delta^{\mathbf{+}}=0$. The findings of this section can be summarized as follows.

Theorem 7. A 3-connected planar map $M$ with $n$ vertices has a convex drawing on a grid of size $\left(n-1-\Delta_{S_{\mathrm{Min}}}^{\oplus}\right) \times\left(n-1-\Delta_{S_{\mathrm{Min}}}^{\oplus}\right)$, where $\Delta_{S_{\mathrm{Min}}}^{\oplus} \geq 0$ is the number of faces with a counterclockwise edge in each color in $S_{\mathrm{Min}}$. Such drawing can be computed in linear time.

\section{Improvements and Limitations}

Our ambition was to design an algorithm for convex drawings of 3-connected planar graphs which at least matches all known algorithms for this task. Theorem 7 shows that we are very close. Still, there is Schnyder's $(n-2) \times(n-2)$ bound for triangulations which is not completely matched by $\left(n-1-\Delta_{S_{\text {Min }}}^{\oplus}\right) \times\left(n-1-\Delta_{S_{\text {Min }}}^{\oplus}\right)$ since there are triangulations with $\Delta_{S_{\text {Min }}}^{-}=0$. An example of such a triangulation is shown in Figure 8.

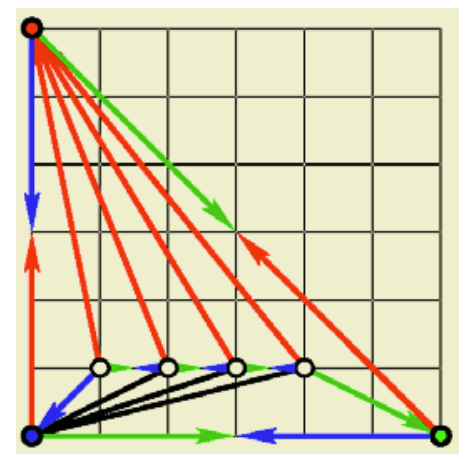

(a)

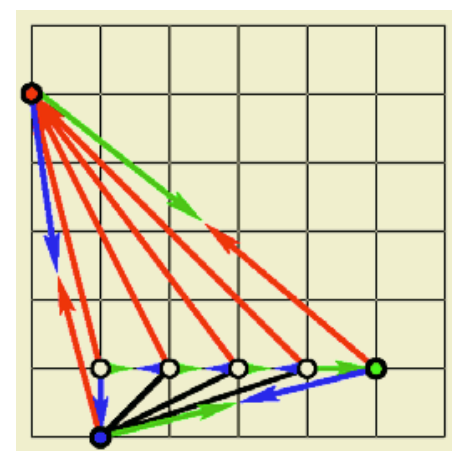

(b)

Fig. 8. (a) A stacked triangulation on the $(n-1) \times(n-1)$ grid. (b) The same graph drawn with the improved method.

It is indeed the case that with any specialization of the algorithm from Section 3 the graph of Figure 8 (a) requires a grid of size $(n-1) \times(n-1)$. 


\subsection{From $n-1$ to $n-2$}

In the standard algorithm, the face-count of the vertices $a_{1}$ is $(f-k-1,0,0)$, $a_{2}$ is $(0, f-k-1,0)$ and $a_{3}$ is $(0,0, f-k-1)$. In order to reduce the grid size, we change the face-count of these two vertices to the following ones: $a_{1}$ is $(f-k-2,0,1), a_{2}$ is $(1, f-k-2,0)$ and $a_{3}$ is $(0,1, f-k-2) .{ }^{3}$ The consequence on the final drawing is the following one: moving $a_{3}$ one unit to the left and one unit to the top, moving $a_{1}$ one unit down and moving $a_{3}$ one unit to the left. Figure 8 (b) gives and example of such drawing.

Using the technique of this section we then obtain:

Theorem 8. A 3-connected planar map $M$ with $n$ vertices has a convex drawing on a grid of size $\left(n-2-\Delta_{S_{\mathrm{Min}}}^{\bullet}\right) \times\left(n-2-\Delta_{S_{\mathrm{Min}}}^{\bullet}\right)$, where $\Delta_{S_{\mathrm{Min}}}^{\oplus} \geq 0$ is the number of faces with a counterclockwise edge in each color in $S_{\mathrm{Min}}$.

\section{Concluding Remarks}

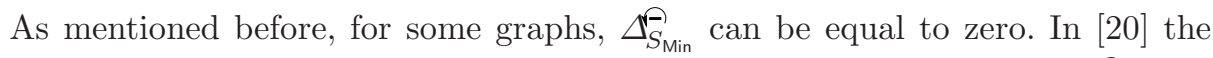
asymptotic average value over $n$-vertices triangulations is given: $E\left(\Delta_{S_{\text {Min }}}\right)=$ $n / 8+o(n)$. Hence the average grid size is significantly lower than the one of the existing algorithms.

\section{References}

1. Rote, G.: Strictly convex drawings of planar graphs (2004)

2. Rosenstiehl, P., Tarjan, R.E.: Rectilinear planar layouts and bipolar orientations of planar graphs. Discrete Comput. Geom. 1 (1986) 343-353

3. Schnyder, W.: Planar graphs and poset dimension. Order 5 (1989) 323-343

4. de Fraysseix, H., Pach, J., Pollack, R.: Small sets supporting Fary embeddings of planar graphs. In: Proc. 20th Annu. ACM Sympos. Theory Comput. (1988) 426-433

5. de Fraysseix, H., Pach, J., Pollack, R.: How to draw a planar graph on a grid. Combinatorica 10 (1990) 41-51

6. He, X.: Grid embeddings of 4-connected plane graphs. Discrete Comput. Geom. 17 (1997) 339-358

7. Miura, K., Nakano, S., Nishizeki, T.: Grid drawings of 4-connected plane graphs. Discrete Comput. Geom. 26 (2001) 73-87

8. Schnyder, W.: Embedding planar graphs on the grid. In: Proc. 1st ACM-SIAM Sympos. Discrete Algorithms. (1990) 138-148

9. Zhang, H., He, X.: Compact visibility representation and straight-line grid embedding of plane graphs. In: Proceedings WADS '03. Volume 2748 of Lecture Notes Comput. Sci., Springer-Verlag (2003) 493-504

10. Tutte, W.T.: Convex representations of graphs. Proceedings London Mathematical Society 10 (1960) 304-320

${ }^{3}$ The modification of the coordinates of $a_{3}$ do not change the grid size. It is done only to give the same role to each suspension vertex. 
11. Tutte, W.T.: How to draw a graph. Proceedings London Mathematical Society 13 (1963) 743-768

12. Kant, G.: Drawing planar graphs using the canonical ordering. Algorithmica 16 (1996) 4-32

13. Chrobak, M., Kant, G.: Convex grid drawings of 3-connected planar graphs. Internat. J. Comput. Geom. Appl. 7 (1997) 211-223

14. Schnyder, W., Trotter, W.T.: Convex embeddings of 3-connected plane graphs. Abstracts of the AMS 13 (1992) 502

15. Di Battista, G., Tamassia, R., Vismara, L.: Output-sensitive reporting of disjoint paths. Algorithmica 23 (1999) 302-340

16. Felsner, S.: Convex drawings of planar graphs and the order dimension of 3 polytopes. Order (2001) 19-37

17. Felsner, S.: Geometric Graphs and Arrangements. Vieweg Verlag (2004)

18. Felsner, S.: Lattice structures from planar graphs. Electron. J. Comb. 11 R15 (2004) 24p.

19. Fusy, E., Poulalhon, D., G.Schaeffer: Coding, counting and sampling 3-connected planar graphs. In: $16^{\text {th }}$ ACM-SIAM Sympos. Discrete Algorithms. (2005) to appear.

20. Bonichon, N., Gavoille, C., Hanusse, N., Poulalhon, D., Schaeffer, G.: Planar graphs, via well-orderly maps and trees. In: Proceedings WG '04. Lecture Notes Comput. Sci., Springer-Verlag (2004) 